\title{
MODELING ECLIPSING BINARIES IN GLOBULAR CLUSTERS
}

\author{
E. F. Milone, ${ }^{1}$ J. Kallrath, ${ }^{2,3}$ C. R. Stagg, ${ }^{1,4}$ and M. D. Williams ${ }^{1}$
}

\section{RESUMEN}

Describimos el uso de las binarias eclipsantes para derivar propiedades fundamentales de las componentes de las binarias en cúmulos estelares. El método requiere el uso de isocronas confiables y de propiedades exactas de las estrellas situadas en éstas.

ABSTRACT
We describe the use of eclipsing binaries to derive fundamental properties of the binary star components in a
cluster. The method requires the use of reliable isochrones and accurate properties of stars that lie along them.

Key Words: BINARIES: ECLIPSING - GALAXIES: CLUSTERS - STARS: FUNDAMENTAL DATA

\section{PROLOGUE, PURPOSE, AND PROBLEM}

The determination of the orbital elements and stellar parameters from light curves usually requires multi-passband and radial-velocity data to resolve ambiguities and insure both high precision and accurate results. However, radial velocities of faint objects are difficult to obtain, especially of systems near the main sequence turn-off in dense clusters; moreover, multiple filter acquisition, desirable for the modeling, sometimes precludes adequate phase coverage in any one passband. In such cases, the assumed and initial guesses of adjusted parameters need to be based on alternative but adequate information. In our present case study, with data in only two passbands, modeling of $H S T$ data of eclipsing binaries in the globular cluster 47 Tuc required the use of information from theoretical isochrones, since spectroscopy was unavailable. Bootstrapping operations were required to produce solutions for the systems, some of which are used to illustrate the technique.

\subsection{Purpose}

The main purpose here is to find the fundamental properties of stars with different metallicities than in the solar neighborhood, viz., in a variety of environments, such as globular clusters, through analyses of eclipsing binaries. Photometry provides the basic data for the determination of the radii and of the distortions due to proximity between the components of the pair, and it is important to discover how these basic properties depend on the chemical

\footnotetext{
${ }^{1}$ RAO, University of Calgary, Calgary, AB, Canada.

${ }^{2}$ BASF, Ludwigshafen, Germany.

${ }^{3}$ Dept. of Astronomy, University of Florida, Gainesville, FL, USA

${ }^{4}$ Joliet Junior College, Joliet, IL, USA.
}

composition. Of particular interest are the masses and radii of stars on the lower part of the main sequence. Accordingly, in this paper we will discuss two such systems in 47 Tuc.

\subsection{Problem}

The definitive determinations of the masses, the scale of the system, and $v_{\gamma}$, which helps to decide membership in a star cluster, all require radial velocities. Even for adequate light curve treatment, the latter are often important to determine which minimum is an occultation and which a transit eclipse.

A major problem in achieving our avowed purpose is that radial velocities are not always available. In such cases, the weight of the solution must be borne primarily by the photometry; this provides a handle on the temperature difference between the components but the absence of a mass ratio makes the extraction a difficult proposition, especially if the stars are well-enough separated so that departure from longitudinal symmetry is minimal.

If the stars are little-enough separated so that Roche geometry is readily perceptible in the light variation outside eclipses, a mass ratio may be determinable (more precisely, able to be modeled), which is why overcontact binaries and even systems with $\beta$ Lyrae-like light curves have become fashionable targets.

For a given system, if we have only one or two light curves and the eclipses are not well covered, we may have a problem to extract the fundamental properties, particularly from the systems that yield potentially the best fundamental data - the longerperiod detached systems. This is especially true for systems with partial eclipses. In such cases, we must have excellent precision in the data so that the shape of the minima is definitive at specific places on the 
rising or falling branches, and at least two of the contact points can be established. These conditions are not always found, so serendipity must play a role, in a field of many targets, in determining which systems can be mined to yield fundamental properties.

For those cases where the light curves are wellenough conditioned to yield high-precision elements, the main problem becomes how we can avoid solution degeneracy when full data sets (multi-passband photometry and radial velocity data) are absent.

\section{SOLUTION}

For radiative properties, two passbands are vastly better than one. The leverage of the two in prying out those properties depends somewhat on the separation of the effective wave numbers of the passbands, and, of course on the relative flux distributions in the components. The relative depths of primary and secondary minimum in the two passbands yields the ratio of the surface brightness in each passband and this ratio is directly related to the colour index difference between the components. Coupled with the system's colour index, each star's temperature is then found, notwithstanding complications introduced by colour excesses due variously to metallicity differences from standards, and interstellar and circumstellar reddening.

Since the photometry provides only the relative size, but the scale of the orbit is required for the absolute size, the radii as well as the masses remain problematic.

In clusters, even in crowded ones, isochrones may allow the removal of this degeneracy. If the isochrones are well matched to cluster observations, the modeling of interstellar extinction and metallicity, if not circumstellar extinction and reddening, is key. These permit complicating phenomena to be treated, even if not uniquely, because particular systems where circumstellar matter or third light may still play a role can very well occur.

With the isochrone models, relative properties of the components are attainable. Exactly how they are extracted is not entirely independent of the tools. We make use of light curve modeling programs that adequately model a system's radiative as well as its geometric and orbital properties.

\subsection{Tools}

The software packages we use are the University of Calgary damped least-squares versions of Wilson's (1998) base program: wd98k93h and wD2002. The basis for and philosophy behind the latter is discussed extensively in Kallrath \& Milone (1999).
Both make use of Kurucz atmosphere models to represent the relative passband fluxes of the component stars at each grid point. The package WD2002 permits auto-iteration of our version of the WilsonDevinney program which we call wd93k93, currently including version $g$. Our latest version of the latter program, $h$, differs from $g$ only in having a larger range of grid sizes available to model eclipses involving a component as small as a Saturn-sized planet. WD2002 permits computation of synthetic light curves and the other options of Wilson's base code, but includes also a simplex program, with which parameter space can be checked for deeper minima than were attained by the base model. The packages can be run consecutively in various combinations, and may be run iteratively. WD2002 runs on PCs with WINDOWS or LINUX operating systems; wd98k93 currently runs under AIX and LINUX.

\subsection{The Initial and Assumed Parameters}

Wilson-Devinney programs use the following parameters from which absolute quantities are ultimately derived:

- semi-major axis, $a$

- eccentricity, $e$

- argument of pericentre, $\omega$

- inclination, $i$

- mass ratio, $q=\mathcal{M}_{2} / \mathcal{M}_{1}$

- passband "luminosity", $\mathcal{L}_{1}(\lambda)$

- period, $P$

- epoch, $t_{0}$

- modified potentials, $\Omega_{1,2}$

- temperature(s), $T_{1,(2)}$

- "third light", $\ell_{3}(\lambda)$

- limb-darkening coefficients, $x_{1,2}(\lambda)$ (and sometimes $y_{1,2}(\lambda)$ for square-root or logarithmic limbdarkening laws), although $x$ coefficients are usually, (and $y$ always) assumed rather than adjusted.

Additionally, in modern Wilson-Devinney codes one may solve for a number of other parameters, such as: the albedos $\left(A_{1,2}\right)$, the first derivatives of $P$ and $\omega$, gravity brightening coefficients $\left(g_{1,2}\right.$, not to be confused with gravitational accelerations), up to two sets of spot parameters (longitude, latitude, angular radius, and temperature factor), a phase shift $\left(\phi_{0}\right)$ to match observational phase values if shifted from 0 at primary minimum mid-eclipse, and the ratio of rotation to revolution factors $\left(F_{1,2}\right)$. Given the tools described in $\S 2.1$, one can properly assume some quantities, such as $x$ (and $y$ ), which depend mainly on $T$ and $\log g$ and have usually only a small dependence on chemical composition. If the light curves are free 
of asymmetries, spots are probably ignorable. Values may be taken for $A_{1,2}, g_{1,2}$ for the relevant (radiative/convective) stellar atmospheres model.

Quantities which are calculated in DC or LC from the parameters include:

- stellar radii for the pole, point, side, and back facings, $r_{\mathrm{p}, \mathrm{pt}, \mathrm{s}, \mathrm{b}}$; and

- gravitational acceleration, $\log g_{1,2}$

- polar surface brightness of each component

- bolometric magnitude of each component

- mass of each component

Given appropriate isochrone models, and a reasonable expectation that at least one component is on the (presumably) single isochrone for the cluster, an initial temperature of that component can be found, along with that star's absolute bolometric and visual magnitudes and mass.

\subsection{Modus Operandi for Obtaining Initial and Unadjusted Parameters}

The operations to achieve the initial parameters are flowcharted in Figures 1 and 2. For present purposes, the depths of the primary and secondary mideclipse minima may be written in classical terms ( $c f$. , Russell \& Merrill 1952):

$d_{p}=1-\ell_{p}=\left(\mathcal{L}_{1}+\mathcal{L}_{2}\right)-\left[\mathcal{L}_{1}+\left(1-\alpha_{0}\right) \mathcal{L}_{1}\right]=\alpha_{0} \mathcal{L}_{1}$

and

$d_{s}=1-\ell_{s}=\left(\mathcal{L}_{1}+\mathcal{L}_{2}\right)-\left[\mathcal{L}_{1}+\left(1-\alpha_{0}\right) \mathcal{L}_{2} k^{2}\right]=\alpha_{0} k^{2} \mathcal{L}_{2} ;$

where $\ell_{\mathrm{p}, \mathrm{s}}$ are normalized light levels, $k$ is the ratio of radii, and $\alpha_{0}$ is the "alpha-function", the eclipsed areal fraction, evaluated at mid-eclipse. The geometric terms are indeed simplified and inexact, but approximately cancel out in the depth ratios.

In a single passband, the depth ratio is:

$$
\frac{d_{\mathrm{p}}}{d_{\mathrm{s}}}=\frac{\mathcal{L}_{1}}{\mathcal{L}_{2} k^{2}}=\sigma_{1} / \sigma_{2},
$$

where $\sigma_{1,2}$ are the passband surface brightnesses.

In these expressions, any differences in limbdarkening and 'reflection' effects between the stars will show up in the surface brightness ratios, which are therefore not quite direct indications of the temperatures integrated over the entire stellar disks. This can be corrected later in the modeling. Given two passbands, the ratio of the depth ratios in each is then:

$$
Q=\frac{\left(d_{\mathrm{p}} / d_{\mathrm{s}}\right)_{\lambda_{1}}}{\left(d_{\mathrm{p}} / d_{\mathrm{s}}\right)_{\lambda_{2}}}=\frac{\left(\sigma_{1} / \sigma_{2}\right)_{\lambda_{1}}}{\left(\sigma_{1} / \sigma_{2}\right)_{\lambda_{2}}}=\frac{\left[\sigma_{1}\left(\lambda_{1}\right)\right] /\left[\sigma_{1}\left(\lambda_{2}\right)\right]}{\left[\sigma_{2}\left(\lambda_{1}\right)\right] /\left[\sigma_{2}\left(\lambda_{2}\right)\right]},
$$

which is directly related to the difference in the colour index, $C_{1,2}$ of the components:

$$
C_{1}-C_{2}=-2.5 \log Q .
$$

So given an initial value of $C_{1}, C_{2}$ is found, and from the corresponding isochrone model, an initial value of $T_{2}$. Modeling analyses with WD2002 can then refine differences in $T$ and $\mathcal{L}$ and may lead to an improvement of $C_{1}$ with the caveat that radiative differences between the stars will cause departures from the effective temperatures of the disks as seen at quadratures or as viewed from above the poles. Of course the light-modeling code adequately corrects for such effects by treating the surface brightness at each grid point with appropriate physics.

The proximity of Star 1 (taken as the star eclipsed at the deeper minimum) to an isochrone point and the deduction of the likely properties of Star 2 from system light and colour suffice to establish starting values of the adjusted and unadjusted parameters. The general procedure is outlined in the flow-charts of Figures 1 and 2, which show, respectively, the earlier and later stages of setting up the DC input files. The steps of Stage 1 are well known to anyone who has done such analyses, but here we are assuming that the light of star 1 dominates the system. Modest resolution spectral information is sometimes available to provide this kind of information, as the GAIA satellite proof of concept series of papers (e.g., Munari et al. 2001; Zwitter et al. 2003) shows, but in this case is not. Some of the steps of Stage 2 may be somewhat less familiar; they provide the main thrust of the method discussed here.

One may begin by assuming that Star 1 properties are those of the system. Then, given $M_{\mathrm{V}}$ and $\left(V-I_{c}\right)_{0}$ and the approximate colour index difference, $\Delta C$, computed from equation (5), $C_{1,2}$ follow. The location of Star 1 on the isochrone can then be checked. After initial modeling trials, the properties of both stars can then be used to check that they continue to fall on the same isochrone or not.

In some cases it is found that only one or neither component falls on the isochrone. Without RV and proper motion data, non-membership in the cluster remains a possibility. If, though, such a system is a member, third light must be contributive to the light of a component lying above the isochrone; this can arise from contamination either by a neighboring 
Modus Operandi (for initial parameters): First Stage

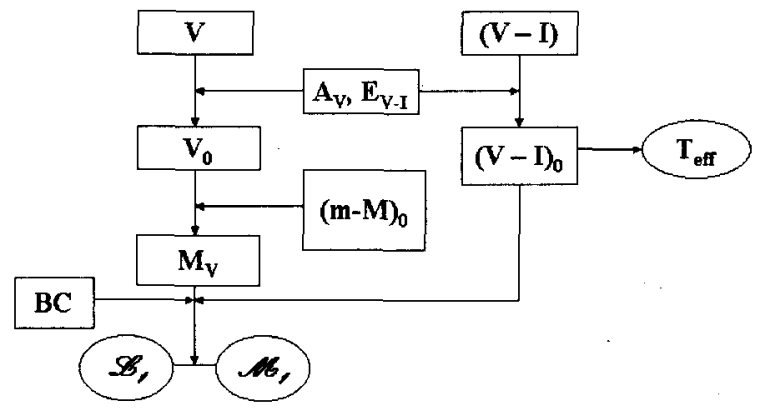

Fig. 1. The first stage of establishing initial and unadjusted parameters. The mid-chart squares contain information from cluster modeling. Star 1 is initially assumed to dominate both light and mass of the system.

\section{Modus Operandi (for initial parameters): Second Stage}

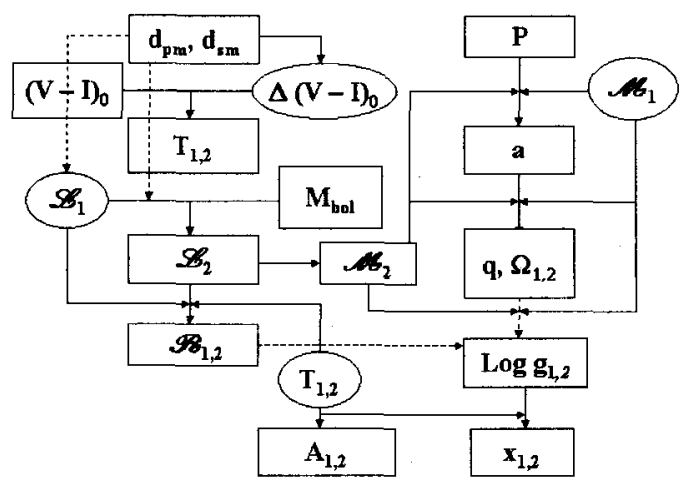

Fig. 2. The later stage of establishing initial and unadjusted parameters.

image in a too-crowded field or by a real companion. An example of the former is discussed in $\S 4$ and an example of the latter is discussed in $\S 3.3$.

Thus by applying the precept that consistency is expected, boot-strapping in this way may be used to establish the properties of both components, and to flag cases where third light should be investigated. The eclipsing binary portion of the 47 Tuc project is at this point.

\section{ECLIPSING BINARIES IN THE GLOBULAR CLUSTER 47 TUC}

The detection of eclipsing variables was a byproduct of a search for transiting planets in this rich globular cluster (Gilliland et al. 2000). The search involved 8.3 days of observation with the Planetary and Wide-Field Cameras aboard the HST.

The number of systems studied thus far is 22 out

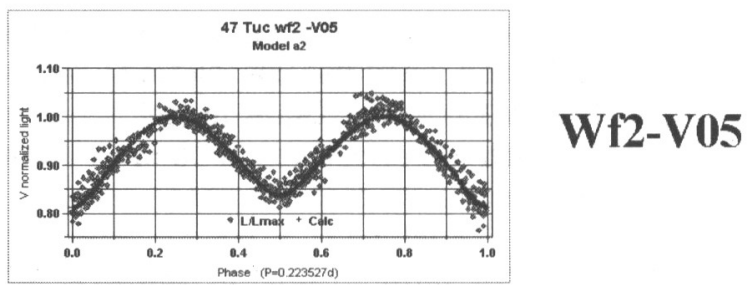

$\mathrm{I}=\mathbf{5 5 . 8 7 ( 4 2 )}$

$\mathrm{T}_{1,2}=4906,4619(27)$

$\Omega_{12}=3.693(17)$

$q=0.9583(69)$

$\mathrm{L} 1(\mathrm{~V})=6.872(101)$

$L 2\left(I_{c}\right)=6.754(81)$

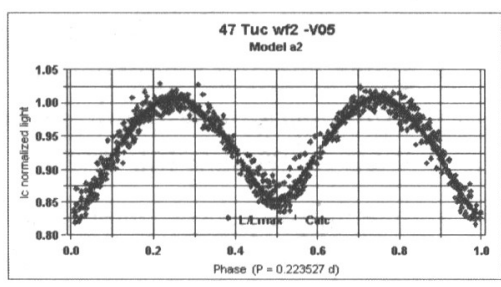

Fig. 3. Two-passband light curve preliminary fittings and converged solution parameters of the over-contact system, WF2-V05.

of more than 30 detected in a field of some 50,000 stars. The isochrone modeling was carried out by D. VandenBerg (private comm., 2000; Bergbusch \& VandenBerg 2001). The adopted cluster model has the following properties:

- $(m-M)_{0}=13.37$

- $E_{V-I_{c}}=0.036$

- $[\mathrm{Fe} / \mathrm{H}]=-0.83$

- $[\alpha / H]=0.3$

- $Y=0.2410$

\subsection{The Eclipsing Systems}

A sample of the systems detected in this rich globular cluster (R. Gilliland, private comm.) is presented in Table 1.

The first column contains the name of the chip and a sequence order number. Some of these were known from prior photometry of the cluster and will not be discussed further here. The table indicates the range of system brightness and colour, as corrected for interstellar extinction and reddening.

\subsection{Tuc Binaries Fittings}

The bulk of the fittings were carried out on a $1 \mathrm{GHz}$ PC running Windows 98; the WD2002 package tasks DC, LC, simplex, and wd98k93g were run auto-iteratively. Final adjustments and checks were carried out with wd98k93g on the University of Calgary's IBM RS6000 running AIX.

The fitted light curves of a low amplitude 0.224-d over-contact system, WF2-V05, can be seen in Figure 3 . 
TABLE 1

PROPERTIES OF A SAMPLE OF 47 TUC ECLIPSING VARIABLES) ${ }^{a}$

\begin{tabular}{lcccccccc}
\hline Variable & $V_{0}$ & $M_{\mathrm{V}}($ obs $)$ & $M_{\mathrm{V}}($ iso $)$ & $\Delta M$ & $\left(V-I_{\mathrm{c}}\right)_{0}$ & $\left(V-I_{\mathrm{c}}\right)($ iso $)$ & $M_{1}($ bol $)$ & $\mathcal{M}_{1}$ \\
\hline PC1-V04 & 17.32 & 3.95 & 4.22 & -0.27 & 0.62 & 0.64 & 4.08 & 0.89 \\
PC1-V07 & 18.66 & 5.29 & 6.15 & -0.85 & 0.85 & 0.85 & 5.91 & 0.72 \\
PC1-V08 & 18.18 & 4.81 & 5.85 & -1.04 & 0.80 & 0.80 & 5.65 & 0.75 \\
PC1-V10 & 17.13 & 3.76 & 3.76 & -0.00 & $\ldots$ & 0.70 & 3.60 & 0.91 \\
PC1-V11 & 17.19 & 3.82 & 2.79 & 1.04 & 0.90 & 0.90 & 2.48 & 0.93 \\
PC1-V21 & 17.40 & 4.03 & 5.40 & -1.37 & 0.73 & 0.73 & 5.23 & 0.79 \\
PC1-V22 & 17.64 & 4.27 & 5.08 & -0.81 & 0.69 & 0.69 & 4.92 & 0.82 \\
WF2-V01 & 17.48 & 4.11 & 5.17 & -1.06 & 0.70 & 0.70 & 5.01 & 0.81 \\
WF2-V02 & 18.85 & 5.48 & 6.15 & -0.66 & 0.85 & 0.85 & 5.91 & 0.72 \\
WF2-V03 & 20.28 & 6.91 & 6.91 & $\ldots$ & 1.41 & 1.02 & 6.52 & 0.65 \\
WF2-V05 & 18.98 & 5.61 & 6.77 & -1.16 & 0.98 & 0.98 & 6.42 & 0.66 \\
WF3-V02 & 19.73 & 6.36 & 7.09 & -0.73 & 1.08 & 1.08 & 6.71 & 0.63 \\
\hline
\end{tabular}

${ }^{\mathrm{a}} V$ and $I_{c}$ data from R. Gilliland and isochrone models from $\mathrm{D}$. VandenBerg (private comm.)

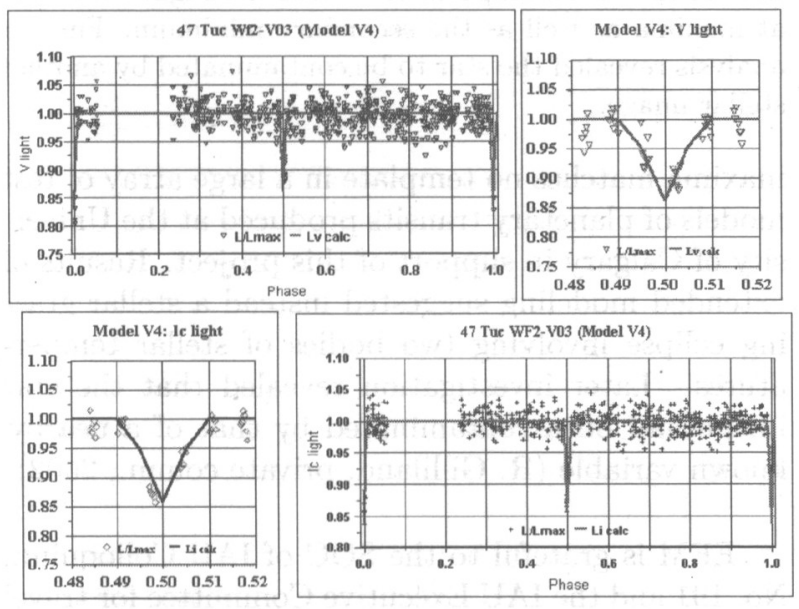

Fig. 4. Two-passband light curve preliminary fittings of the system WF2-V03, with secondary minima fittings in the two passbands also shown.

\subsection{Tuc Binaries Solutions}

Preliminary fittings for WF2-V03 and the fittings and solution for WF3-V02 obtained with the WD2002 package are shown in Figures 4 and 6 . The solution for WF2-V03 is characterized by the details provided in Figure 5.

WF2-V03 appears to be a 10.09-d period Algol system, with very red, low mass components.

WF3-V02 appears to be a 0.414 -d period $\beta$-Lyrae system.

The placements of these systems and their components are shown on the color-magnitude diagram
Wf2-V03 Solution

\begin{tabular}{|c|c|c|c|}
\hline Parameter & Value & m.s.e. & Correction \\
\hline $\mathrm{i}$ & $88 . \overline{1380}$ & 0.0647 & 0.0142 \\
\hline $\mathrm{T} 2$ & 0.4136 & 0.0013 & -0.0003 \\
\hline Omega 1 & 33.2230 & 0.6539 & 0.2603 \\
\hline Omega 2 & 34.3950 & 1.4609 & 0.0590 \\
\hline to & 2451360.7708 & 0.0017 & 0.00042 \\
\hline$P$ & 10.0911 & 0.0025 & -0.0004 \\
\hline $\mathrm{Ll}(\mathrm{V})$ & 7.8483 & 0.3656 & -0.0160 \\
\hline $\operatorname{Li}(x)$ & 7.6985 & 0.3610 & -0.0224 \\
\hline
\end{tabular}

Sum( $\left.W^{\star} \operatorname{Res}^{\star \star} 2\right)$ for input values:

sigma (avge wt): 14.864946

0.017656

Fig. 5. The WF2-V03 parameters of the converged solution. The passband luminosities, $L 1(V, B)$, are in units of $4 \pi$.

for 47 Tuc, Figure 7. All the target stars plus a handful of non-variable stars and previously known systems are shown on the $M_{V}$ vs. $\left(V-I_{\mathrm{c}}\right)_{0}$ isochrone. The results of the analyses of two systems are also shown. The components are plotted with respect to both ordinates. WF2-V03 is the reddest of the binaries, and the less massive component of this system falls beyond the reliable limits of the $\mathcal{M}$ vs. $\left(V-I_{\mathrm{c}}\right)_{0}$ isochrone curve.

Note that all four components appear to have normal masses (if the mass models extrapolate cleanly to the low secondary mass of the WF2-V03 secondary) but neither the primary nor the sec- 


\section{Wf3-V02 Fittings}
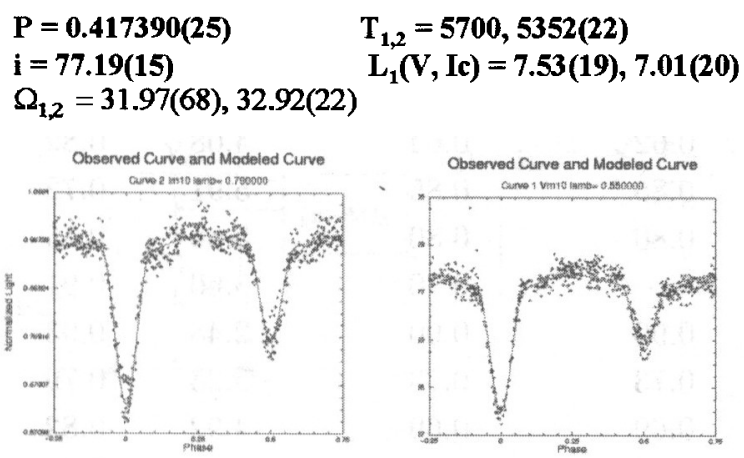

Fig. 6. Preliminary initial, assumed, and computed properties of WF3-V02.

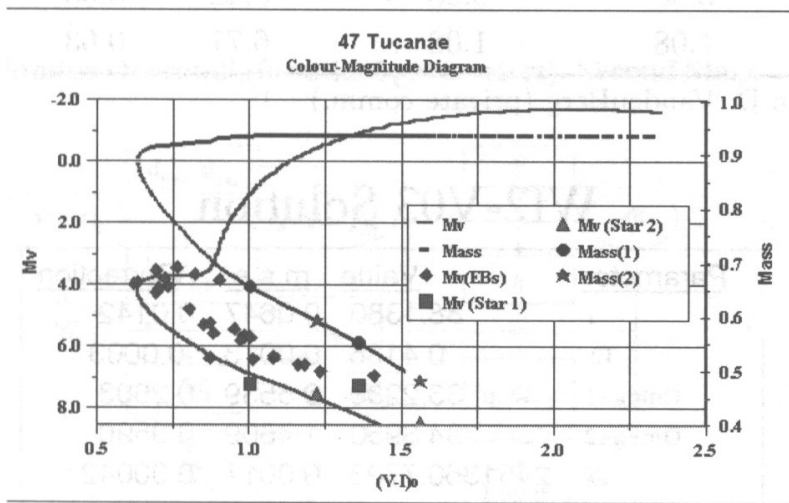

Fig. 7. The Colour-Magnitude Diagram for 47 Tuc, courtesy of Don VandenBerg. The results of analyses of the systems WF2-V03 and WF3-V02 are shown.

ondary of this system fits the isochrone well. This system is therefore a candidate for third light modeling. In contrast, the components of the Wf3-V02 system fall on the CMD isochrone and its masses agree well with the models and both its components appear on the the cluster's mass vs. colour-index relation.

\section{POSTSCRIPT}

The light curve modeling procedure yields reasonably satisfactory results in nearly all cases we have worked on, but some of the low amplitude variations may be due to rotation of spotted stars or to pulsation, rather than to grazing stellar eclipses.

The HST experiment with 47 Tuc found only one planetary transit candidate, WF2-V02. However, the light curve fittings seen in Figure 8 demonstrate that the low amplitude primary minimum is unlikely to be due to a planetary transit since the presence of a secondary minimum and the slight curvature in the

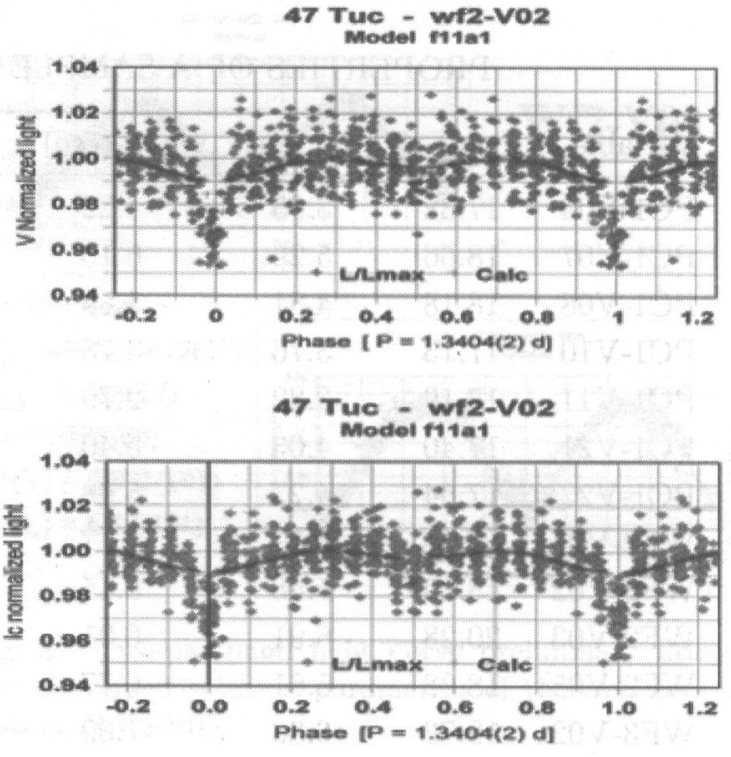

Fig. 8. Light curves of the sole planetary transit candidate in the 47 Tuc experiment. Note the slight curvature at maxima as well as the secondary minimum. Further analysis revealed the star to be contaminated by another stellar image.

maxima matches no template in a large array of test models of planetary transits produced at the University of Calgary in support of this project. Results of extended modeling suggested instead a stellar grazing eclipse involving two bodies of stellar temperatures. Later investigation revealed that the star image had been contaminated by that of a nearby, known variable (R. Gilliland, private comm., 2002).

EFM is grateful to the SOC of IAU Colloquium No. 191 and the IAU Executive Committee for travel support. The work described in this paper was supported by NSERC of Canada and by the University of Calgary Research Grants Committee. Limbdarkening coefficients were provided by $\mathrm{W}$. Van Hamme and a desktop package to facilitate interpolation among temperature and $\log g$ for fixed values of $[\mathrm{Fe} / \mathrm{H}]$ was provided by Dirk Terrell.

\section{REFERENCES}

Bergbusch, P. \& VandenBerg, D. A. 2001, ApJ, 556, 322 Gilliland, R. L. et al. 2000, ApJ, 545, L47

Kallrath, J. \& Milone, E. F. 1999, Eclipsing Binary Stars: Modeling and Analysis. (New York: Springer-Verlag New York, Inc)

Munari, U., Tomov, T., Zwitter, T., Milone, E. F., Kallrath, J., Marrese, P. M., Boschi, F., Prsa, A., Tomasella, L., \& Moro, D. 2001, A\&A, 378, 477

Russell, H. N., \& Merrill, J. E. 1952, Princeton Observa- 
tory Contributions, 26

Wilson, R. E. 1998, Computing Binary Star Observables (Reference Manual to the Wilson-Devinney Program). (Gainesville, FL: Department of Astronomy,
University of Florida)

Zwitter, T., Munari, U., Marrese, P. M., Prsa, A., Milone, E. F., Boschi, F., Tomov, T., \& Siviero, A. 2003, A\&A, 404, 333

\section{DISCUSSION}

Scarfe - How far out in the cluster were you working?

Milone - The main frames were in the crowded core. The idea was to find two dozen planets!

Scarfe - So you had to put up with lot of contamination!

Milone - That's the worry!

E. F. Milone \& M. D. Williams: Dept. of Physics \& Astronomy, University of Calgary, 2500 University Dr., N.W., Calgary, AB T2N 1N4, Canada (milone, williamd@ucalgary.ca).

Josef Kallrath: BASF-AG, Scientific Computing (GVC/S-B009), D67056 Ludwigshafen, Germany; \& Dept. of Astronomy, University of Florida, Gainesville, FL 32611, USA (josef.kallrath@t-online.de).

C. R. Stagg: Joliet Junior College, 1215 Houbolt Rd., Joliet, IL 60431, USA (cstagg@sbcglobal.net). 